https://dx.doi.org/10.35366/96462

\title{
Resúmenes de Trabajos Libres
}

Free Papers Abstracts

Resumo dos trabalhos livres

\author{
Síndrome de Takotsubo asociado con \\ COVID-19: reporte de un caso \\ Galván Merlos María Alaciel, Rangel Vargas Uriel Emmanuel, \\ Vera Aguirre Guadalupe, Díaz Ramírez Abraham, \\ Arellano Ramírez Alfredo \\ Hospital Regional de Alta Especialidad Ixtapaluca.
}

Se ha observado que COVID-19, además de otros órganos, también afecta al corazón. Las formas graves de COVID-19 pueden producir un aumento de troponina, no necesariamente debido a un síndrome coronario agudo, pero podrían asociarse con formas no isquémicas como miocarditis, miocardiopatía por estrés y coagulopatía. Takotsubo es un síndrome clínico caracterizado por una disfunción reversible del ventrículo izquierdo ( $\mathrm{VI})$, a menudo es relacionado con un evento estresante emocional o físico que precede al síndrome o incluso sin un desencadenante en algunos casos. El patrón típico de anomalía regional del movimiento de la pared del VI es abombamiento apical con hipercinesia basal. En raras ocasiones, también se ha observado que Takotsubo se desarrolla después de infecciones del tracto respiratorio (aunque la tasa de incidencia no está clara) y $10 \%$ de los casos progresa a choque cardiogénico. Presentamos a un hombre de 53 años de edad con factores de riesgo cardiovasculares y neumonía por COVID-19. Se observó que tenía un infarto agudo al miocardio, se le realizó cateterismo cardiaco sin encontrarse lesión vascular y se reportó como hallazgo área aneurismática apical, por lo cual se diagnosticó miocardiopatía de Takotsubo. En nuestro paciente la isquemia aguda se atribuye a un estado procoagulante secundario, estasis y daño endotelial por COVID-19.

\section{Cardiopatía por estrés secundaria a COVID-19}

Carrillo Ramírez Silvia, Martínez Monter Julisa, Teniza Frías Eduardo, Enríquez Barajas Adriana, Solís González Jesús Hospital Ángeles Pedregal.

Introducción: La miocardiopatía por estrés se caracteriza por una depresión transitoria de la función contráctil del ventrículo izquierdo con compensación de la pared basal ventricular. Objetivo: Presentar un caso y revisión de la literatura acerca de la cardiomiopatía por estrés secundaria a COVID-19. Caso clínico: Femenino de 46 años ingresa por estado general, fiebre, tos y diarrea, se aplica prueba de SARS-CoV-2 que resulta positiva. Inicia terapéutica con hidroxicloroquina y azitromicina, que se suspende por alargamiento de intervalo QT. Cuatro días después de su ingreso a terapia intensiva con evento de dolor precordial tipo anginoso y taquicardia sinusal de hasta 150 latidos por minuto, por lo que se solicita curva enzimática que comienza a elevarse desde la segunda toma y ecocardiograma, el cual muestra miocardiopatía por estrés. Se inicia tratamiento con antiagregación plaquetaria, estatinas e inotrópico del tipo levosimendán; el cuadro se remite de manera adecuada. Discusión: Se ha comentado en varios reportes de la literatura que el SARS-CoV-2 tiene efectos a diversos niveles; pese a que la paciente no desarrolló un cuadro de deterioro respiratorio franco tuvo deterioro cardiaco secundario a COVID-19, por lo que consideramos de manera relevante hacer la documentación del caso.

Prevalencia de eventos adversos asociados a terapia anticoagulante en pacientes con fibrilación auricular e infección por SARS-CoV-2
Chávez Requena Irina, Aguirre Sánchez Janet,

Franco Granillo Juvenal

Centro Médico Hospital ABC, Ciudad de México.

Universidad Nacional Autónoma de México.

Introducción: Los procesos infecciosos generan respuesta inflamatoria sistémica compleja como parte de la inmunidad innata, activan la coagulación y generación de trombina como componentes críticos de comunicación entre las vías de amplificación humoral y celular, lo que se llama inmunotrombosis. La evidencia sobre la coagulopatía y riesgo de trombosis en pacientes con infección por COVID-19 es cada vez mayor; sin embargo, existe poca evidencia sobre el manejo en pacientes con COVID-19 y fibrilación auricular (FA). Objetivo: Evaluar factores de riesgo y complicaciones en pacientes con diagnóstico de FA e infección por COVID-19. Material y métodos: Estudio retrospectivo del 01 de febrero al 31 de junio del 2020. Se incluyeron 25 pacientes con diagnóstico de fibrilación auricular y COVID-19 tratados con anticoagulación. Se evaluó la prevalencia de pacientes COVID-19 y FA, factores de riesgo protrombóticos, estado inflamatorio, terapia farmacológica y complicaciones. Resultados: Niveles elevados de dímero D, IL-6, ferritina y cuenta de plaquetas se asocian a sangrado. De la muestra, $8 \%$ presentó úlcera gástrica, $4 \%$ epistaxis, $4 \%$ hematoma retroperitoneal, $4 \%$ hemorragia de tubo digestivo alto, $4 \%$ hemorragia de tubo digestivo bajo y $68 \%$ no tuvieron complicaciones. Conclusión: Pacientes con FA y COVID-19 que además presentan niveles elevados de dímero $D$, IL-6, ferritina y plaquetas representan un reto terapéutico, ya que presentan mayor riesgo de sangrado contra la necesidad de recibir tromboprofilaxis por patología de base.

\section{Características clínicas de pacientes con choque} cardiogénico y COVID-19 en el Departamento de Medicina Crítica «Dr. Mario Shapiro» del Centro Médico ABC Villalobos Álvarez Víctor Hugo, Franco Granillo Juvenal, Aguirre Sánchez Janet

Centro Médico ABC, Ciudad de México.

Introducción: El choque cardiogénico (ChC) es una entidad grave descrita en el contexto de cardiopatía isquémica; la incidencia mundial es de $4 \%$ y su mortalidad de $45 \%$. Existen casos de ChC secundario a SARS-CoV-2, con mortalidad de 75\%. En México se desconocen las características de estos pacientes. Objetivo: Describir las características clínicas y epidemiológicas en pacientes con ChC y COVID-19. Material y métodos: Estudio observacional y descriptivo en una cohorte de pacientes hospitalizados en la Unidad de Cuidados Intensivos (UTI) entre el 13 de marzo y el 31 de mayo de 2020. Se analizaron datos demográficos y mortalidad. Resultados: Fueron 10 pacientes, edad de 65 años ( $D E \pm 7.34$ ), $60 \%$ hombres, $60 \%$ con diabetes mellitus tipo 2 (DM2) y $30 \%$ con hipertensión arterial sistémica, índice de masa corporal de 30.63 ( $\mathrm{DE} \pm 6.01)$, delta $\mathrm{CO}_{2}$ arteriovenoso $7.7 \mathrm{mmHg}(\mathrm{RIC}=5.9-11.57)$, NT-proBNP $3,549 \mathrm{pg} / \mathrm{dL}(\mathrm{RIC}=755.7-17313)$, el inotrópico mayormente utilizado fue levosimendán (90\%); APACHE II de 14 (RIC = 9-27), SOFA de 9 (RIC = 5-15.5), estancia en la UTI de 28 días $(\mathrm{RIC}=18-39.7)$; la falla orgánica múltiple estuvo presente en $90 \%$, mortalidad de $60 \%$. Conclusión: El ChC tiene alta mortalidad en pacientes con COVID-19. En nuestro estudio la mayoría padecían sobrepeso y DM2. La causa del ChC en estos pacientes se desconoce, probablemente fueron de origen multifactorial; además, $100 \%$ tenía diagnóstico de sepsis, esto pudiera ser un factor relevante para ser tomado en cuenta. 
Prevalencia de infección pulmonar por Aspergillus spp. en pacientes con neumonía por SARS-CoV-2 tratados con tocilizumab en el Departamento de Medicina Crítica «Dr. Mario Shapiro»

Gómez-de-Segura González Daniel, Franco Granillo Juvenal, Aguirre Sánchez Janet Silvia

Centro Médico ABC Campus Observatorio. Ciudad de México, México.

Introducción: Con la pandemia por COVID-19, la lesión pulmonar ha incrementado, así como la presencia de Aspergillus spp. en algunos reportes de caso. Conocer la prevalencia en relación al uso de inmunomoduladores es esencial. Objetivo: Conocer la prevalencia de Aspergillus spp. en pacientes con COVID-19 en el Departamento de Medicina Crítica tratados con tocilizumab. Material y métodos: Estudio retrospectivo descriptivo en casos con COVID-19 ingresados en junio de 2020 tratados con tocilizumab, quienes presentaron infección por Aspergillus, siguiendo su evolución hasta el alta hospitalaria. Se implementó el protocolo CAPA (COVID-19 Associated Pulmonary Aspergillosis) para el diagnóstico. Resultados: Fueron 52 sujetos, 47 recibieron tocilizumab. El $42.55 \%$ tenía diabetes mellitus. Se encontró una prevalencia de CAPA de $19.1 \%(n=9)$. La dosis acumulada de tocilizumab fue $737 \mathrm{mg}(400-1,120)$. Los días en la Unidad de Terapia Intensiva (UTI) 24.2 (11-38) y días de ventilación mecánica invasiva (VMI) 12.2 (5-23). Discusión: Nuestra prevalencia de CAPA es similar a otros estudios, pero no hay reportes de tratados con tocilizumab. Los días de estancia en la UTI/VMI y dosis de tocilizumab fueron mayores en la población con CAPA.

\section{Manejo exitoso de terapia de soporte de órganos extracorpóreos (ECOS) en neumonía por COVID-19: reporte de caso \\ Villasana Salazar Cynthia Estefanía, Ortiz López Cynthia Isabel, Rizo Topete Lilia María, Hernández Portales Jorge Alberto Hospital Christus Muguerza Alta Especialidad, Monterrey, Nuevo León.}

Los pacientes con neumonía severa por COVID-19 pueden desarrollar síndrome de distrés respiratorio agudo, lo que desencadena falla respiratoria. Las terapias de soporte de órganos extracorpóreos (oxigenación por membrana extracorpórea [ECMO], terapia de reemplazo renal continuo [CRRT], plasmaféresis) se pueden utilizar como parte del manejo adyuvante en los pacientes con hipoxemia refractaria aun con el uso adecuado de ventilación mecánica óptima y de medidas farmacológicas. Se describe el caso de un hombre de 31 años quien a su ingreso se maneja con ventilación mecánica invasiva (VMI) y por mala evolución se decide inicio de terapia ECMO dentro de las primeras 24 horas de su ingreso; posteriormente, se complementa manejo con CRRT con filtro oXiris y plasmaféresis en los primeros tres días de estancia intrahospitalaria. El paciente presenta una rápida mejoría clínica y se observa una disminución importante en marcadores inflamatorios. La CRRT con filtro oXiris se ha asociado con una disminución significativa en niveles de citoquinas y endotoxina; mientras que la terapia con plasmaféresis elimina también mediadores inflamatorios. El manejo con ECMO ha mostrado beneficios como terapia de rescate en pacientes con hipoxemia. El éxito de estas terapias depende en gran medida del inicio temprano de ECOS en el paciente crítico.

Morbilidad y mortalidad de pacientes obstétricas en una Unidad de Cuidados Intensivos de un hospital de segundo nivel de atención

Hernández López Guillermo David, Escobar Ortiz Diana, Valenzuela Plata Alfredo, Arellano Guevara Daniel,

Luna Cuéllar Antonio

UMAE Magdalena de las Salinas. Instituto Mexicano del Seguro Social.

Objetivo: Determinar la morbilidad y mortalidad de las pacientes obstétricas admitidas en una Unidad de Cuidados Intensivos de un hospital de segundo nivel de atención. Material y métodos: Estudio descriptivo, retrospectivo y observacional efectuado en pacientes obstétricas que ingresaron a la Unidad de Cuidados Intensivos (UCl) del Hospital General de Atizapán en enero de 2019. Se analizaron las siguientes variables: características maternas, aspectos relacionados con el embarazo, parto y puerperio, días de ventilación mecánica, estancia en la UCl y mortalidad materna. Resultados: Se registraron 57 ingresos. Los trastornos hipertensivos del embarazo (38.5\%), la hemorragia obstétrica $(15.7 \%)$ y la insuficiencia respiratoria aguda $(12.2 \%)$ fueron los principales motivos de ingreso. Treinta y cinco por ciento de las pacientes requirió ventilación mecánica. El ultrasonido pulmonar mostró predominio de patrón intersticial focalizado en $73.6 \%$ de las pacientes. En cinco pacientes (8.7\%) se encontró aumento del diámetro de la vaina del nervio óptico, en un caso se correlacionó con la presencia de edema cerebral mediante tomografía de cráneo. Se registraron cinco muertes maternas. Conclusiones: Más de $80 \%$ de los ingresos ocurrieron durante el postparto; las pacientes multíparas (45.6\%) tuvieron mayor tasa de ingreso a la Unidad de Cuidados Intensivos, mayor grado de obesidad y más complicaciones.

\section{Mortalidad en pacientes con COVID-19 sometidos a terapia de reemplazo renal (TRR) en el Departamento de Medicina Crítica «Dr. Mario Shapiro» del Centro Médico ABC \\ Cruz Bolaños Hans de Jesús, Aguirre Sánchez Janet Silvia, Franco Granillo Juvenal \\ Centro Médico ABC. Ciudad de México, México.}

Introducción: La incidencia de lesión renal aguda en pacientes con infección por SARS-CoV-2 es del 10-20\%, de los cuales el 2 al $22 \%$ reciben terapia de reemplazo renal. Al momento se ha reportado una incidencia de 50 a $100 \%$ de mortalidad en este tipo de sujetos. Objetivo: Determinar la mortalidad en pacientes sometidos a terapia de reemplazo renal (TRR) con infección por SARS-CoV-2. Material y métodos: Estudio observacional descriptivo retrospectivo del 1 de abril al 31 de agosto de 2020 en el Departamento de Medicina Crítica «Dr. Mario Shapiro» del Centro Médico Hospital ABC. Resultados: Fueron 357 pacientes, $26(7.2 \%)$ requirieron TRR, 61\% hombres, edad media de 53 años (48-62), días promedio de TRR 14 (4-22). La mortalidad fue de $45 \%$. En el análisis multivariado, no se encontraron variables predictivas para mortalidad en las variables sociodemográficas, diabetes tipo $2 \mathrm{OR}=0.25$ (IC 95\% 0.11-12.4, $\mathrm{p}=0.83$ ), hipertensión arterial OR = 1.31 (IC 95\% 0.292-50.83, $p=0.30)$ y enfermedad cardiovascular previa $\mathrm{OR}=1.73(\mathrm{IC} 95 \% 0.719-42, \mathrm{p}=0.10)$. Conclusión: Nuestra mortalidad coincide con la mortalidad reportada en la literatura internacional, lo cual nos lleva a mejorar estrategias diagnósticas y terapéuticas para evitar la progresión de la lesión renal.

\section{Terapia de reemplazo renal continua en lesión} renal aguda asociada con embarazo y puerperio en terapia intensiva de un hospital de tercer nivel

Cardona Trabado Catherine Esther,

Zaragoza Galván José de Jesús, Cerón Díaz Ulises Wilfrido,

Moguel González Bernardo

Hospital Español de México.

Introducción: La lesión renal aguda (LRA) asociada al embarazo y puerperio continúa siendo un reto diagnóstico y una de las principales causas de morbimortalidad materna y perinatal. Objetivo: Determinar la tasa de recuperación de función renal en pacientes obstétricas con LRA, sometidas a terapia de reemplazo renal continua (TRRC) en la Unidad de Terapia Intensiva (UTI) de un hospital de tercer nivel. Material y métodos: Estudio observacional, retrospectivo y descriptivo. Se recolectaron 99 pacientes del 2009 al 2020; incluyéndose siete con seguimiento de la función renal por creatinina y tasa de filtrado glomerular (TFG) a 30 días. Se emplearon medidas de tendencia central y t de Student. Resultados: La principal indicación de TRRC fue alteración ácido-base severa (tiempo mínimo 34 horas y máximo 255 , promedio $111.57 \pm 69$ ). Las patologías obstétricas relacionadas al desarrollo de LRA fueron: HELLP (71.42\%), choque hipovolémico $(57.14 \%)$ y ruptura hepática $(42.85 \%)$. La función renal a 30 días 
mostró mejoría significativa en la creatinina $(p=0.0223)$ y la TFG $(p=$ 0.042). Discusión: Aproximadamente $40 \%$ de la LRA durante el embarazo se asocia con enfermedad hipertensiva del embarazo. Existe poca experiencia en el tratamiento de la LRA mediante TRRC durante el embarazo y puerperio, incrementando el riesgo de disfunción orgánica múltiple y modificando el pronóstico.

Cetoacidosis euglucémica como complicación en infección por COVID-19

Rivera Saldaña Rosa Emilia, Guzmán Ramírez Perla Marlene, Márquez Valdez Manuel Alejandro, Carrillo Ramírez Silvia del Carmen, Esponda Prado Juan Gerardo

Hospital Ángeles Pedregal.

Introducción: Se ha puesto poca atención a las alteraciones metabólicas que son producidas por COVID-19, especialmente en los portadores de diabetes mellitus (DM). Objetivo: Ejemplificar las complicaciones metabólicas en pacientes infectados por COVID-19, resultando en áreas inciertas para el diagnóstico y tratamiento. Caso clínico: Masculino de 49 años con DM2 en tratamiento con empagliflozina/linagliptina más pioglitazona/metformina refiriendo adecuado control. Inició el 13 de abril de 2020 con faringitis, odinofagia, tos y disnea progresiva, recibió antibiótico sin mejoría, con empoderamiento del cuadro. Se recibió con Sat 70\%, FR $36 \times$ min, acidosis metabólica de anión gap elevado. La tomografía axial computarizada (TAC) de tórax presentó imágenes compatibles para infección por COVID-19. Requirió hemodiálisis convencional sin mejoría de la acidosis metabólica. Se encuentra cetonuria y cetonas en suero, por lo que se inicia infusión de insulina. Por no presentar mejoría se inicia terapia de sustitución renal en modalidad lenta, lo cual mejora el equilibrio ácido base. Una vez suspendida la infusión de insulina, con adecuada tolerancia, manteniendo equilibrio ácido-base y resto de paraclínicos con mejoría de parámetros de inflamación, se decide su egreso de la UCI. Discusión: La acidosis refractaria como complicación metabólica en pacientes con COVID-19 pasa desapercibida al no ser una manifestación respiratoria típica en la infección.

Asociación del índice neutrófilo-linfocito

con mortalidad en COVID-19

Sarabia Collazo Ángel Antonio, Muñoz Ramírez María del Rosario, Chávez Pérez Carlos Eduardo, Sánchez Nava Víctor Manuel Hospital San José TecSalud.

Antecedentes: El índice neutrófilo/linfocito (INL) es un biomarcador de disfunción endotelial con valor pronóstico en enfermedades cardiovasculares, infecciosas y oncológicas. Sin embargo, su utilidad en COVID-19 no ha sido demostrada. Objetivo: Evaluar asociación del índice INL como factor pronóstico de mortalidad en pacientes COVID-19. Material y métodos: Estudio retrospectivo, observacional, en pacientes ingresados del 01 al 31 de julio a la Unidad de Cuidados Intensivos del HSJ TecSalud. Se analizaron variables demográficas y analíticas. Se definió el INL como el cociente entre los valores absolutos de neutrófilos y linfocitos. La variable de desenlace fue mortalidad. Tipo de análisis estadístico inferencial de comparación de medias (ANOVA). Resultados: Se incluyeron 200 pacientes, con edad media de 55 años (23-92 años de edad, mediana de 55), de los cuales $74.2 \%$ eran de género masculino, con una mortalidad general de $27 \%$ de la muestra. El INL en análisis de varianza de un solo factor muestra una significancia estadística $(p<0.05)$, en la que se observa una mortalidad mayor en la población con índice Neu/Linf menor al momento de ingreso a unidad cuidados intensivos adultos (UCIA). Conclusiones: El INL es un biomarcador de fácil acceso que tiene valor como factor pronóstico de mortalidad en COVID-19.

\footnotetext{
Muerte materna secundaria a hipertensión intracraneana por EVC hemorrágico

Enríquez Barajas Adriana, Teniza Frías Eduardo, Pacheco Aispuro Gerónimo, Carrillo Ramírez Silvia del Carmen, Martínez Monter Julisa Hospital Ángeles Pedregal.
}

El aumento de la presión intracraneana (PIC) es factor pronóstico relacionado con mortalidad en pacientes neuroquirúrgicos. La hipertensión intracraneana (HIC) se define como una elevación sostenida de la PIC por encima de sus valores normales, secundaria a la pérdida de los mecanismos compensatorios o ruptura del equilibrio existente entre el cráneo y su contenido. Se presenta el caso de paciente femenino de 37 años con antecedente de puerperio mediato. Inicia con deterioro neurológico y crisis convulsivas. La tomografía muestra hemorragia subaracnoidea Fisher IV. Angiografía diagnóstica con ausencia de seno lateral derecho como variante anatómica. Se realiza ventriculostomía izquierda y colocación de catéter de presión intracraneal. Medidas antiedema sin respuesta y evoluciona a muerte cerebral. A pesar del desarrollo tecnológico, los métodos invasivos siguen siendo el estándar de oro para el monitoreo de la PIC. La ventriculostomía permite obtener un registro y sirve como una válvula de liberación de presión al permitir el drenaje de líquido cefalorraquídeo. Los métodos quirúrgicos para el control de la PIC deben ser considerados, en pacientes específicos, como parte del tratamiento primario.

Lesión cerebral traumática en adultos mayores:

epidemiología, resultados e implicaciones futuras

Hernández López Guillermo David, Escobar Ortiz Diana,

Gorordo Delsol Luis Antonio, Vargas Rentería Sharon Adlahi, Luna Cuéllar Antonio

UMAE Magdalena de las Salinas. Instituto Mexicano del Seguro Social.

Introducción: La lesión cerebral traumática (LCT) surge en la actualidad como una nueva epidemia silenciosa entre los adultos mayores. Objetivo: Identificar las principales causas de LCT y conocer la mortalidad en este grupo de pacientes. Material y métodos: Se incluyeron todos los adultos mayores que ingresaron con diagnóstico de traumatismo craneoencefálico durante el periodo comprendido entre abril y septiembre de 2020. Resultados: Los adultos mayores tienen las tasas más altas de hospitalización y muerte relacionadas con el traumatismo cerebral. Las caídas fueron la principal causa de LCT (58\%), los accidentes de tránsito son el segundo (17.2\%) y las agresiones representan $6.8 \%$ de las LCT. La edad avanzada y el tiempo de evolución del trauma influyen negativamente en el resultado después de un traumatismo craneoencefálico (TCE). El sexo masculino se asoció con una mayor incidencia de lesión cerebral traumática (TBI, por sus siglas en inglés) en los adultos mayores (71.42\%), el sexo femenino no se asocia con mejores resultados. La mortalidad general fue de $51.7 \%$. Discusión: Los adultos mayores con LCT experimentan una mayor morbilidad y mortalidad, trayectorias de recuperación más lentas y peores resultados funcionales, cognitivos y psicosociales. En los adultos mayores la LCT está mayormente relacionada con caídas, la mortalidad es alta a pesar del tratamiento intensivo.

\section{Encefalitis asociada a COVID-19}

Olguín Ramírez Leticia Alejandra, Ramírez García Héctor Alejandro, Chávez Pérez Carlos Eduardo, Sánchez Nava Víctor Manuel Staff UCIA COVID, Hospital San José TecSalud, Tecnológico de Monterrey.

Introducción: Las complicaciones neurológicas asociadas a COVID-19 se presentan en 5-80\% de los casos. Los síntomas principales son encefalopatía, cefalea, vértigo, neuropatía, entre otros. Objetivo: Describir la aparición de una respuesta inflamatoria secundaria neurológica en un paciente con COVID-19 crítico. Material y métodos: Se realizó análisis retrospectivo del expediente clínico y estudios de imagen. Resultados: Masculino 51 años, hipertenso y diabético. Ingresa a UTIA por SIRA severo; requirió ventilación mecánica, desarrolló lesión renal aguda (LRA) AKI-III, taquicardia supraventricular y choque séptico. Se suspende sedación a los 10 días de intubación. Al séptimo día posterior a suspensión de sedación con FOUR Score 5 (E1 M0 B4 R0). Presenta nistagmo horizontal y vertical continuo, inagotable, ROTS aumentados generalizados, mioclonías faciales. En la RMN de cerebro se documentan hiperintensidades en T2 Flair con restricción en difusión en centros semiovales, corona radiada y 
periventricular. Punción lumbar con pleocitosis, PCR y IgG SARSCoV-2 negativo, IL-6 elevada, proteína básica de mielina y bandas oligoclonales presentes. Enolasa sérica negativa. Se administran cinco bolos de esteroide, terapia anticomicial e inmunoglobulina. RMN de control al mes sin lesiones en corona radiada y centros semiovales. Conclusión: La respuesta inflamatoria secundaria a COVID-19 puede ser documentada en LCR con marcadores inflamatorios (IL-6, FNT, IL1, ACE).

\section{Daños neurológicos en neumonía severa}

por SARS-CoV-2: reporte de un caso

Ramos Margarito Samuel, Gerardo Morales Ana Deysi,

Martínez Blanca Camelia

Star Médica Centro.

Introducción: En la nueva enfermedad respiratoria causada por el coronavirus 2019 y su síndrome severo SARS-CoV-2 se aprecia que hasta $34 \%$ de los pacientes presentaron diversos síntomas neurológicos; algunas investigaciones mencionan desmielinización, neurodegeneración y senescencia celular. Objetivos: Reporte de un caso con neumonía grave por SARS-CoV-2 y daño neurológico. Caso clínico: Masculino de 34 años con obesidad GII, inicia el primero de junio de 2020 con disnea, fiebre y taquipnea, saturación de $45 \%$ medio ambiente, tomografía axial computarizada (TAC) con daño severo en cuatro cuadrantes, se intuba con ventilación protectiva, sedoanalgesia, miorrelajante, prono, saturación irregular. Leucocitos 14,500, DD 1,796 , ferritina 1,882. Se complica con sepsis pulmonar por Acinetobacter baumannii y es tratado con meropenem, linezolid. Se le realizó traqueostomía por ventilación prolongada a los 20 días. Sin sedantes se observa indiferente al medio, fuerza 1/5, ROTS disminuido, la resonancia magnética nuclear (RMN) muestra cambios en base de núcleos corticales precentral, polos occipitales en mesencéfalo, cambios asociados con encefalopatía hipóxico-isquémicas, por lo cual se realiza rehabilitación física. Resultados: Consideramos que los cambios neurológicos del paciente son secundarios directamente al COVID-19. Discusión: Existen pocos reportes de secuelas neurológicas por COVID-19, se mencionan volúmenes agrandados de la corteza olfativa, hipocampo, ínsulas, opérculo. Siempre debemos vigilar en esta esfera a los pacientes. A los 90 días el paciente se vale por sí mismo.

\section{Neumomediastino espontáneo en paciente}

con COVID-19. Reporte de caso clínico

Fraire Félix Irving Santiago, Varela Martínez Eliseo,

Hermosillo Ulloa Mariana Janeth, Castillo González Roberto Alejandro, Villavicencio Bautista Juan Carlos

Centenario Hospital Miguel Hidalgo (CHMH), Aguascalientes, Aguascalientes.

Introducción: El neumomediastino espontáneo se define como la presencia de aire o gas en el mediastino de forma primaria. Su etiología puede ser primaria o secundaria. Fue definida como síndrome clínico específico en 1819 por Laennec. Caso clínico: Paciente femenino de 33 años sin antecedentes personales patológicos, se diagnostica PCR positivo para COVID-19. Se decide intubación orotraqueal por persistencia de falla respiratoria y se ingresa a la Unidad de Cuidados Intensivos. Durante su estancia se identifica enfisema subcutáneo en región de cuello y tórax, por lo que se realiza tomografía axial computarizada (TAC) de tórax que evidencia neumomediastino. Valorada por el Servicio de Cirugía de Tórax y Otorrinolaringología, se realiza traqueostomía y colocación de sonda mediastinal anterior, su evolución es tórpida y fallece por choque séptico refractario. Discusión: El neumomediastino espontáneo (NE) es un síndrome clínico caracterizado por la presencia de aire en el espacio mediastínico sin que exista una causa traumática o quirúrgica previa. Su incidencia real no está establecida. El NE es una complicación muy rara de las neumonías víricas. Se han publicado algunos casos aislados asociados con la neumonía por SARS (síndrome respiratorio agudo severo), la neumonía por virus influenza A (H1N1) y la neumonía por SARS-CoV-2.
Aspergilosis como complicación en el tratamiento de COVID-19 Guzmán Ramírez Perla Marlene, Márquez Valdez Manuel Alejandro, Carrillo Ramírez Silvia del Carmen, Rivera Saldaña Rosa Emilia, Esponda Prado Juan Gerardo

Hospital Ángeles Pedregal.

Introducción: Al buscar la disminución de la respuesta inflamatoria sistémica, los agentes inmunosupresores permiten vulnerabilidad a infecciones oportunistas que complican el escenario clínico. Objetivo: Describir el caso de aspergilosis posterior a la intervención de inmunosupresores. Caso clínico: Masculino de 39 años de edad, ingresa por dificultad respiratoria tras iniciar siete días previos con cuadro de infección de vías respiratorias bajas; tratamiento previo con oseltamivir. Presentó hemoptisis desde su ingreso a la UTI, se inició tratamiento con metilprednisolona, azitromicina, kaletra, oseltamivir, meropenem. Se cuenta con imagen sugestiva para aspergilosis pulmonar y galactomanano positivo, además de PCR positivo. Presentó hemorragia alveolar difusa, se completó tratamiento con voriconazol y anfotericina B. Requirió traqueostomía por presentar ventilación mecánica prolongada. Conclusiones: Aunque se cuente con amplia cobertura antimicrobiana en los pacientes con COVID-19 bajo tratamiento inmunosupresor se debe tener en cuenta la posibilidad de infección por oportunistas, ya que éstos pueden ser un obstáculo para la evolución.

Falsos negativos en PCR SARS-CoV-2 y un diagnóstico exacto Enríquez Barajas Adriana, Teniza Frías Eduardo,

Rivera Saldaña Rosa Emilia, Esponda Prado Juan,

Carrillo Ramírez Silvia del Carmen

Hospital Ángeles Pedregal.

COVID-19, enfermedad infecciosa causada por el virus SARS-CoV-2, se propaga fácilmente. La mayoría de las personas con COVID-19 presenta enfermedad respiratoria de leve a moderada y es posible que algunas cursen asintomáticas, mientras que otras experimentan síntomas graves y precisan de un tratamiento especializado. El diagnóstico formal requiere análisis de laboratorio o pruebas de diagnóstico por imagen. Femenino de 81 años, hipertensa y antecedente de contacto con persona positiva para COVID-19, inicio de cuadro infeccioso de vías respiratorias que evoluciona a neumonía atípica y falla respiratoria grave. Prueba PCR SARS-CoV-2 negativa en tres ocasiones y tomografía de tórax con imágenes de neumonía multisegmentaria en vidrio despulido sugestivas de COVID-19. La primera información diagnóstica, y la más accesible, es la que proviene de los signos y síntomas de la exploración clínica, la cual se complementa con estudios de laboratorio y gabinete. La identificación exacta de las personas infectadas permite que se les dé un tratamiento oportuno además de prevenir la propagación de la infección. La sensibilidad de las pruebas depende del tiempo de evolución del padecimiento. El diagnóstico exacto debe ser un complemento entre los signos y síntomas que presenta el paciente y los resultados de los estudios de laboratorio y gabinete.

Utilidad de la escala HACOR para predecir falla de la ventilación mecánica con puntas nasales de alto flujo Ibarra Ibzan Salvador, Pizaña Dávila Alejandro Hospital Ángeles Mocel.

Introducción: La ventilación no invasiva (VNI) se usa con frecuencia en pacientes con insuficiencia respiratoria hipoxémica. Su tasa de fracaso permanece alto (25-59\%), lo que indica que no todos los pacientes se benefician con este tratamiento. Objetivo: El objetivo primario de este estudio es determinar la utilidad de la escala HACOR como predictor de fracaso de las puntas nasales de alto flujo (PNAF) en el síndrome de insuficiencia respiratoria aguda secundaria a neumonía viral por COVID-19. Material y métodos: Se realizó un estudio retrospectivo, analítico, observacional en la Terapia Intensiva del Hospital Ángeles Mocel, se realizó análisis de $\chi^{2}$. Resultados: Se obtuvieron datos de una muestra de 30 pacientes de los cuales. Se encontró que 
un puntaje HACOR de cinco puntos o más a las dos horas de inicio de la ventilación no invasiva con PNAF tiene una sensibilidad de $84 \%$ y una especificidad de 81\%, AUC: 0.82 (IC 95\% 0.67-0.98) p > 0.05. Discusión: Algunos expertos han publicado que los esfuerzos inspiratorios espontáneos vigorosos pueden conducir rápidamente a una lesión pulmonar autoinducida por el paciente (LPAP), por lo que la ventilación mecánica debe emplearse de manera temprana para evitar la progresión de una lesión leve a una lesión pulmonar más grave.

\section{Beneficio del decúbito prono prolongado en pacientes ventilados con síndrome de insuficiencia respiratoria aguda moderada a severa debida a enfermedad por coronavirus 2019 Turrubiates Hernández Theno Alexandro, * \\ Ramírez Gutiérrez Álvaro Eduardo \\ *Medicina Crítica. ${ }^{*}$ Hospital Regional de Ciudad Madero, Petróleos Mexicanos.}

Introducción: Se desconoce el beneficio de extender el tiempo de decúbito prono mayor a 24 horas en pacientes ventilados con síndrome de distrés respiratorio agudo (SDRA) por la enfermedad por coronavirus 2019 (COVID-19). Objetivo: Conocer el beneficio del decúbito prono prolongado en pacientes ventilados por COVID-19. Material y métodos: Estudio observacional y retrospectivo de pacientes ventilados con SDRA por COVID-19 y decúbito prono mayor a 24 horas, en el periodo de abril a septiembre de 2020. Se registraron horas de duración, muerte, puntuaciones SOFA y MuLBSTA. Se agruparon de acuerdo con la duración. Resultados: Se incluyeron 30 pacientes. Se dividieron en dos grupos: A en prono $>72$ horas $(n=8)$ y $B$ en prono $<72$ horas $(n=22)$. Las puntuaciones se reportaron en medianas; SOFA, grupo A de 4.5 y grupo B de 6 ( $p$ 0.447), MuLBSTA en el grupo A de 7.5 y de 8 en el grupo $B$ ( $p$ 0.344). Con relación a muerte, el grupo A reportó $75 \%(n=6)$ y en el grupo $B, 68.2 \%(n=$ 15) (p 0.547). Discusión: No se observó significancia estadística con relación a muerte entre ambos grupos. El prono prolongado, como medida de mejoría de los niveles de oxigenación, puede beneficiar a los pacientes ventilados con COVID-19.

\section{Asfixia traumática. Reporte de caso}

Sosa Medellín Miguel Ángel, ${ }^{*}$ Ruvalcaba Ayala Daniel, ${ }^{*}$

Rentería Oloño Diana, ${ }^{\ddagger}$ Del Real Rodríguez Pedro Antonio de Jesús ${ }^{\ddagger}$ *Unidad Médica de Alta Especialidad No. 21, IMSS. Monterrey, N.L.

¥Unidad Médica de Alta Especialidad No. 25, IMSS. Monterrey, N.L.

Presentamos el caso de un paciente masculino de 50 años de edad, quien cuenta con antecedentes de hipertensión arterial sistémica, diabetes mellitus tipo 2 en manejo con fármacos vía oral y obesidad. Ingresa al Servicio de Urgencias de la Unidad Médica de Alta Especialidad No. 21 al participar en un accidente en el que es aplastado por un vehículo automotor (camión de carga) sobre pared de forma aguda. Se identifican lesiones en tórax, fracturas costales y hemoneumotórax bilateral. Además, desarrolla cambios de coloración del tórax, cuello y cara de aspecto violáceo, petequias en tórax y cuello, así como cambios de coloración a rojizo de las conjuntivas (característica de asfixia traumática [AT]). La AT es una condición rara subdiagnosticada, se genera por compresión brusca del tórax y abdomen, caracterizándose por lesiones descritas previamente. La asfixia es causada por la compresión de venas del abdomen que ocasiona distensión de la vena cava superior, tributaria y capilar lo que produce atonía capilar con o sin ruptura vascular afectando cabeza, cuello y cara, probablemente por incompetencia valvular. El tratamiento está enfocado a las lesiones asociadas (en este caso sonda intrapleural); su pronóstico dependerá de las lesiones que se desarrollen en tórax y en abdomen.

\footnotetext{
Asociación del poder mecánico e intensidad ventilatoria con la mortalidad en pacientes con COVID-19 grave

Hernández Pedroza José Carlos, ${ }^{*}$ Pinedo Lechuga Marcela ${ }^{\ddagger}$ *Medicina del Enfermo en Estado Crítico.

¥CMN Noreste UMAE No. 25, IMSS, Monterrey.
}

Introducción: El poder mecánico (PM) y la intensidad ventilatoria (IV) se han asociado con daño pulmonar inducido por ventilador. Objetivo: Evaluar la asociación del PM y la IV con la mortalidad en pacientes con COVID-19 grave. Material y métodos: Estudio observacional, de cohorte, retrospectivo realizado en pacientes con COVID-19 grave. Resultados: Se incluyeron 56 pacientes de edad media $57.9 \pm$ 11.7 años (46.4\% femeninos/53.6\% masculinos). La IV no fue distinta entre fallecidos y sobrevivientes, pero el PM fue significativamente mayor en fallecidos. La mortalidad global fue de $60.7 \%$; en pacientes con un $\mathrm{PM}>15 \mathrm{~J} / \mathrm{min}$ fue de $91.7 \%$ y en aquellos con un $\mathrm{PM}<15$ $\mathrm{J} / \mathrm{min}$ al primer día fue de $52.3 \%(\mathrm{p}=0.018)$. El RR para mortalidad con un PM > $15 \mathrm{~J} / \mathrm{min}$ fue de 10.0 (IC 95\%, 1.2-84.6, p = 0.018). En el análisis multivariado el PM se asoció independiente y significativamente a mortalidad. El área bajo la curva ROC del PM para predecir mortalidad fue de 0.627 , de la IV fue de 0.527 , de la puntuación APACHE II fue de 0.721 y de un modelo combinado de PM + APACHE II fue de 0.774. Conclusiones: Un PM $>15 \mathrm{~J} / \mathrm{min}$ se asoció significativamente con mayor mortalidad en pacientes con COVID-19 grave y en combinación con el puntaje APACHE-II al ingreso mejora su capacidad predictiva.

Intoxicación por dióxido de cloro como prevención de neumonía por SARS-CoV-2: a propósito de un caso Esparza Mendoza Alejandra, Lara Cruz Juan,

Lira González Julio César, Herrera Morales Blanca Estela HGR No. 196, IMSS.

Introducción: Hasta el momento no se conocen agentes preventivos ni tratamientos curativos contra el SARS-CoV-2, de manera comercial el dióxido de cloro, aunque es una sustancia altamente reactiva provocando irritación en boca, esófago y estómago, pueden presentarse náuseas, vómito, diarrea y trastornos cardiovasculares y renales. Caso clínico: Mujer de 19 años, quien ingresa procedente de urgencias con el diagnóstico de choque no especificado, postoperada de laparotomía (LAPE) por apendicitis grado I, con persistencia de inestabilidad hemodinámica. Después de la ingesta de $10 \mathrm{~mL}$ de dióxido de cloro mezclado con $1,000 \mathrm{~cm}^{3}$ de solución de manera profiláctica ante SARS-CoV-2, presenta intolerancia a la vía oral, náusea, vómito, diarrea, acrocianosis con presencia de oximetría de $50 \%$ y dolor abdominal; se realiza apendicectomía, presentando deterioro neurológico y compromiso respiratorio, por lo que se inicia manejo avanzado de la vía aérea. Se realiza TAC de tórax, lo cual expone neumonía probablemente compatible con SARS-CoV-2 y por rezonificación se decide envío a nuestra unidad médica. Presenta fiebre, inestabilidad hemodinámica, lesión renal, edema agudo pulmonar, desequilibrio hídrico electrolítico, elevación de transaminasas y anemia. Después de seis días, se realiza extubación de la vía aérea sin aparentes complicaciones, a la exploración neurológica con FOUR 16 pts, se realiza TAC simple de cráneo sin complicaciones.

\section{Utilización de oxigenoterapia de alto flujo en pacientes con} COVID-19 con hallazgos graves por tomografía computarizada Rangel Vargas Uriel Emmanuel, ${ }^{*}$ Galván Merlos María Alaciel, ${ }^{*}$ Herrera Morales Blanca Estela, ${ }^{\ddagger}$ Vera Aguirre Guadalupe ${ }^{\ddagger}$ *Medicina Crítica. ${ }^{\ddagger}$ Hospital General Regional No. 196 «Fidel Velázquez Sánchez». Hospital Regional de Alta Especialidad Ixtapaluca.

La oxigenoterapia de alto flujo (OAF) permite una mejoría considerable en la oxigenación a través de distintos mecanismos, además de tener efectos benéficos a nivel hemodinámico. En la actualidad, la atención del enfermo en estado crítico por neumonía COVID-19 ha colapsado los sistemas de atención en salud a nivel mundial, y nuestro país no es la excepción. El OAF es un recurso limitado en la mayoría de unidades hospitalarias del sector salud; sin embargo, su inclusión podría disminuir la necesidad masiva de camas hospitalarias y reducir la estancia de pacientes en unidades de cuidados intensivos al evitar la necesidad de ventilación mecánica, además de evitar sus complicaciones asociadas. Motivo por el cual se presenta una serie 
de casos conformada por cinco pacientes con neumonía por SARSCoV-2, que presentaron insuficiencia respiratoria aguda requiriendo incremento gradual de oxígeno suplementario a pesar de lo cual persistieron con hipoxemia severa, ingresando a Unidad de Cuidados Intensivos del Hospital Regional de Alta Especialidad de Ixtapaluca, siendo manejados desde su ingreso al servicio con OAF, cursando con evolución favorable sin requerir de ventilación mecánica durante su estancia a pesar del mal pronóstico, de acuerdo con los hallazgos graves documentados por tomografía computarizada a su ingreso.

Relación de la oxigenación en prono despierto como predictor de ventilación mecánica invasiva

Cruz Ruiz Néstor Gabriel, Tapia Pastrana Gabriela, González Rosas Rolando, Santiago Arenas Keyla HGZ No. 1 «Dr. Demetrio Mayoral Pardo» del Instituto Mexicano del Seguro Social, Oaxaca.

Introducción: El síndrome de distrés respiratorio agudo asociado con COVID-19 (CARDS) ocurre en 20-40\%. La posición prona mejora la oxigenación y reduce la mortalidad de 32.8 a $16 \%$ en no COVID-19 con SIRA. La mejoría de la oxigenación es debida a mejoría de la relación ventilación-perfusión del reclutamiento de las porciones dorsales. La posición prona en pacientes no intubados mejora la oxigenación, reduce el esfuerzo respiratorio, la lesión pulmonar y la necesidad de intubación. Objetivo: Buscar un valor predictivo con relación a cambios de la oximetría al realizar maniobra de pronación para intubación mecánica. Material y métodos: Pacientes con diagnóstico de COVID-19 por reacción en cadena de la polimerasa (PCR), sin ventilatoria mecánica, catalogados con SIRA moderados a severo sin contraindicaciones para realizar maniobra de posición prona, identificar el momento indicado para intubación basado en el monitoreo de la oximetría. Resultados: Se realiza estudio observacional, transversal, retrospectivo, en pacientes que mejoraban la saturación más de $4 \%$ de su valor basal al realizar pronación se evitaba intubación al menos 48 horas. Discusión: Los cambios de valores de oximetría a la maniobra de pronación en pacientes no intubados podrían identificarse al momento para realizar intubación. Se requiere de estudio con mayor número de pacientes para buscar significancia estadística.

Rehabilitación física un reto frente a pacientes con neumonía grave por infección secundaria a SARSCoV-2 en la Unidad de Cuidados Intensivos Adultos Dircio Nava Juan, ${ }^{*}$ Lara Cruz Juan,,

Herrera Morales Blanca Estela, ${ }^{\ddagger}$ Melchor García Pedro§ "Medicina del Enfermo en Estado Crítico. $¥$ Unidad de Cuidados Intensivos. §Hospital General Regional No. 196 «Fidel Velázquez», Instituto Mexicano del Seguro Social.

México se encuentra en un proceso de transición, transformación e investigación en salud. Derivado de la pandemia generada por el virus SARS-CoV-2, los tratamientos y protocolos establecidos en la medicina actual se replantean tomando nuevos criterios, lo que representa nuevos desafíos y establece parámetros basados en la evidencia. Los pacientes con neumonía grave por infección secundaria SARS-CoV-2 tienen un alto riesgo de desarrollar alteraciones funcionales. Desencadenados por la necesidad de ventilación mecánica durante periodos prolongados, la sedación profunda, el bloqueo neuromuscular, la necesidad de presión positiva al final de la espiración alta y la inmovilidad prolongada afecta la musculatura en general, poniendo al paciente en riesgo para desarrollar debilidad adquirida en las Unidades de Cuidados Intensivos y/o disfunción diafragmática inducida por ventilación mecánica. Dentro de las intervenciones generales de fisioterapia para pacientes con COVID-19 en la fase aguda, se encuentran principalmente la movilidad oportuna (temprana) y la fisioterapia respiratoria. En nuestro país no existen investigaciones o propuestas de trabajo terapéutico centradas en el movimiento en los pacientes críticos que presentan COVID-19; por esta razón, se propone realizar estudios de casos describiendo el proceso de rehabilitación a pacientes de la Unidad de Cuidados Intensivos Adultos del Hospital General Regional No. 196.
Complicaciones trombóticas pulmonares en pacientes con ventilación mecánica invasiva por COVID-19 en el Departamento de Medicina Crítica «Dr. Mario Shapiro» del Centro Médico ABC Espinosa-Aznar Eduardo, Franco-Granillo Juvenal,

Aguirre-Sánchez Silvia Janet, Chaires Gutiérrez Rodrigo Centro Médico ABC. Ciudad de México, México.

Introducción: Los pacientes con afectación pulmonar grave por COVID-19 presentan hipoxia y aumento progresivo del dímero D, por lo que los síntomas se sobreponen con la embolia pulmonar. En este trabajo se revisaron los hallazgos clínicos, bioquímicos y de imagen de tromboembolismo pulmonar en pacientes con ventilación mecánica invasiva y enfermedad por COVID-19. Objetivo: Determinación de la incidencia de eventos trombóticos pulmonares en pacientes con ventilación mecánica invasiva y afectación pulmonar grave por COVID-19 en el Departamento de Medicina Crítica «Dr. Mario Shapiro». Material y métodos: Estudio retrospectivo descriptivo de pacientes con ventilación mecánica invasiva y diagnóstico de COVID-19 en la Unidad de Cuidados Intensivos, se realizó una angiotomografía pulmonar para descartar o confirmar la existencia de embolismo pulmonar. Se calcularon las medias y rangos de las variables cuantitativas y cualitativas en forma de proporciones. Resultados: Se realizaron 22 estudios de angiotomografía de arterias pulmonares a 212 pacientes con ventilación mecánica invasiva y COVID-19 entre el 15 de marzo y el 27 de agosto de 2020. Se estudió un total de 22 pacientes (16 hombres y seis mujeres) con una edad comprendida entre los 50 y los 75 años y una media de 62.5 años. Se utilizó la escala de Wells para probabilidad intermedia de embolia pulmonar con promedio de 6.2. Se evidenció la existencia de embolia pulmonar en 18 pacientes. En 12 casos se observó afectación en ramas lobares y segmentarias. Se reportó defunción de seis casos a los que se les realizó angiotomografía pulmonar. Discusión: La tromboembolia pulmonar es una complicación de la enfermedad por COVID-19. El pronóstico es variable de acuerdo con los antecedentes y la presentación clínica del paciente en estado crítico.

Decúbito prono prolongado en pacientes ventilados con síndrome de distrés respiratorio aguda moderada a severa por enfermedad por coronavirus 2019

Turrubiates Hernández Theno Alexandro, *

Ramírez Gutiérrez Álvaro Eduardo ${ }^{\ddagger}$

*Medicina Crítica. $¥$ Hospital Regional Cd. Madero, PEMEX.

Introducción: Se desconoce el beneficio de extender el tiempo de posición decúbito prono mayor de 24 horas en pacientes ventilados con síndrome de distrés respiratorio agudo (SDRA) moderado a severo por la enfermedad por coronavirus 2019 (COVID-19). Objetivo: Comparar la mortalidad en decúbito prono prolongado $>72$ horas vs prono $<72$ horas en pacientes ventilados por COVID-19. Material y métodos: Estudio retrospectivo de pacientes ventilados con SDRA por COVID-19 para comparar mortalidad de dos grupos: (a) prono $>72$ horas vs (b) prono < 72 horas; de abril a septiembre de 2020. Resultados: Se incluyeron 30 pacientes en dos grupos: (a) prono $>72$ horas $(n=8)$ y $(b)$ prono $<72$ horas $(n=22)$. Medias de poder mecánico inicial: grupo (a) 25.38 (DE \pm 3.81 ) y grupo (b) 22.55 (DE $\pm 2.70)$ ( $p$ 0.410). Delta presión inicial: grupo (a) $22.38(\mathrm{DE} \pm 8.8)$ y grupo (b) 21.09 ( $D E \pm 6.1$ ) (p 0.196). Medianas de SOFA inicial: grupo (a) de 4.5 (RIC 2.25-6) y grupo (b) de 6 (RIC 2.7-9) (p 0.447). La mortalidad en el grupo (a) fue de $75 \%(n=6)$ y del grupo (b) $68.2 \%(n$ =15) ( $p$ 0.547). Discusión: No se observó diferencia en mortalidad entre los grupos de posición prono $>72$ horas $\mathrm{y}<72$ horas.

\section{Tromboembolia pulmonar aguda y \\ COVID-19: reporte de un caso}

Galván Merlos María Alaciel, * Vera Aguirre Guadalupe, ${ }^{\ddagger}$

Arellano Ramírez Alfredo§

*Medicina Crítica. ¥Unidad de Cuidados Intensivos.

$\S$ Hospital Regional de Alta Especialidad Ixtapaluca. 
La infección por SARS-CoV-2 representa un desafío tanto a los recursos de los servicios sanitarios como a la capacidad para realizar diagnósticos adecuados a causa de la amplia gama de complicaciones, entre las cuales la más amenazadora es la insuficiencia respiratoria aguda grave. Sin embargo, existen informes sobre otras complicaciones potencialmente mortales, como la tromboembolia pulmonar provocada por la COVID-19. La coagulopatía ocurre comúnmente en la sepsis y puede predecir los resultados en la COVID-19 grave. El colapso alveolar, la vasoconstricción pulmonar hipóxica y la ventilación mecánica aumentan la resistencia vascular pulmonar y, en consecuencia, la carga del ventrículo derecho. Dos diferencias importantes respecto al SDRA clásico pueden reducir el riesgo de insuficiencia del VD en la infección por SARS-CoV-2: a) la disminución de la vasoconstricción pulmonar hipóxica, y b) la mayor distensibilidad pulmonar. Ambos factores, junto con un mayor riesgo de fenómenos tromboembólicos, hacen que la tromboembolia pulmonar sea un diagnóstico muy probable cuando aparece insuficiencia del ventrículo derecho en un paciente infectado, independientemente de las características radiográficas. Son necesarios nuevos estudios para definir los criterios clínicos que permitan seleccionar los pacientes con mayor riesgo de TEP, con infección por SARS-CoV-2.

Infecciones asociadas con COVID-19 en pacientes de Cuidados Intensivos (UCI)

Ortiz Martínez Karina, Franco Granillo Juvenal, Aguirre Sánchez Janet Silvia

Centro Médico ABC Campus Observatorio. Ciudad de México, México.

Introducción: Los pacientes en la UCI tienen un alto riesgo de desarrollar infecciones nosocomiales o neumonías asociadas con ventilación mecánica. Los estudios realizados en pacientes con COVID-19 son escasos; sin embargo, se ha mencionado en un estudio post mortem que la coinfección se presenta en el $50 \%$. Además, se reporta que el $8 \%$ de pacientes en la $\mathrm{UCl}$ han presentado coinfecciones fúngicas o bacterianas, los microorganismos más frecuentes son Staphylococcus aureus y Acinetobacter baumannii. Objetivo: Describir infecciones asociadas con COVID-19 en la UCI. Material y métodos: Estudio observacional, descriptivo, retrospectivo, del 17 de agosto al 25 de septiembre de 2020, en la UCl del Centro Médico ABC «Dr. Mario Shapiro". Se analizaron pacientes con diagnóstico confirmado para SARS-CoV-2, con cultivo de secreción bronquial positivo. Resultados: De 30 pacientes, el 67\% fueron hombres, edad media de 61 años, $56.6 \%$ padecía hipertensión arterial y $46.6 \%$ diabetes mellitus 2. En el $78 \%$ (19 casos) de intubados, se aisló Pseudomona aeruginosa en 50\%, Stenotrophomonas maltophilia 20\% y Aspergillus 15\%. Conclusiones: La Pseudomona aeruginosa fue el agente más frecuente en nuestra $\mathrm{UCl}$, difiriendo con lo reportado en la literatura, lo cual da pie al desarrollo de estudios futuros para determinar los factores que llevaron a este resultado.

Asociación de trombosis y niveles de marcadores inflamatorios en pacientes con neumonía grave por SARSCoV-2 (COVID-19) que están con anticoagulación López Reymundo Paulo Sergio, Aguirre Sánchez Janet Silvia, Franco Granillo Juvenal

Centro Médico ABC Campus Observatorio. Ciudad de México, México.

Introducción: El endotelio tiene un papel clave en la respuesta inmunológica, con mayor susceptibilidad a infecciones virales. La fisiopatología de la coagulopatía asociada con COVID-19 afecta al pulmón en una fase temprana, posteriormente progresa a una respuesta inflamatoria sistémica regulada por marcadores inflamatorios que causan lesión endotelial generando trombosis. Objetivo: Evaluar la asociación de trombosis y niveles de marcadores inflamatorios en pacientes con neumonía grave por SARS-CoV-2 con anticoagulación. Material y métodos: Estudio de cohorte, analítico, retrospectivo y longitudinal, se incluyeron pacientes que ingresaron en el periodo de mayo-junio a la unidad COVID del Departamento de Medicina Crítica «Dr. Mario
Shapiro", con diagnóstico de neumonía grave por SARS-CoV-2. Se revisaron expedientes electrónicos para obtener los marcadores inflamatorios. Se realizó un análisis lineal de mediciones repetitivas por prueba de ANOVA utilizando el programa SPSS. Resultados: Setenta y ocho por ciento fueron hombres, $20 \%$ presentó trombosis a pesar de estar con anticoagulación. Se obtuvo significancia estadística para interleucina-6 p 0.019 y PCR p 0.030. Discusión: La interleucina-6 y PCR fueron marcadores inflamatorios asociados con trombosis. Leucocitos, plaquetas, PCT y ferritina carecen de sensibilidad predictiva para trombosis en pacientes con neumonía grave por SARS-CoV-2.

Interleucina 6, dímero D y plaquetas y su correlación con mortalidad, severidad de presentación y ventilación mecánica en pacientes críticos con infección por SARS-CoV-2

Castro Serrano Alejandro, Sánchez Calzada Armando,

Franco Granillo Juvenal, Aguirre Sánchez Janet Silvia,

Ochoa Ramírez Carlos Alberto

Centro Médico ABC Campus Observatorio. Ciudad de México, México.

Introducción: El recuento plaquetario es un marcador asociado con severidad y mortalidad. Un mayor nivel de dímero $D$, interleucina 6 y trombocitopenia se asocian con mayor severidad de presentación de SIRPA y mortalidad. Objetivo: Correlacionar niveles de plaquetas, IL6 , DD vs gravedad, ventilación mecánica invasiva (VMI) y mortalidad en COVID-19. Material y métodos: Cohorte retrospectiva descriptiva en pacientes críticos con COVID-19 del 1 de abril al 30 de junio del 2020. Resultados: 37 casos, $64.9 \%$ hombres, comorbilidades en $54.1 \%$, mortalidad $18.9 \%$. Ventilación mecánica invasiva en hombres y mujeres en 40.5 y $18.9 \%$. Días de estancia en la UCI 14 y 10.6 días. Días de ventilación mecánica 6.5 y 5.3 días. La IL-6 (332 \pm 332 vs $728 \pm 534[p=0.014])$ y la trombocitopenia $(162 \pm 82$ vs $99 \pm 32[p=$ $0.05]$ ) se asociaron con mortalidad al día uno y siete. EI DD correlacionó con gravedad por SOFA ( $r=0.38[p=0.019])$, los pacientes que requirieron $\mathrm{VMI}$ cursaron con mayores niveles de DD al ingreso (658 \pm 224 vs $1,802 \pm 1,499$ [p $=0.006])$. La trombocitopenia se asoció a menor $\mathrm{PaO}_{2} / \mathrm{FiO}_{2}(\mathrm{r}=0.37$, [ $\left.\mathrm{p}=0.022]\right)$. Conclusión: La medición de IL-6, DD y plaquetas son marcadores útiles al ingreso y día siete para predecir severidad de presentación en COVID-19.

Absceso cerebral. Reporte de un caso

Carrillo Ramírez Silvia del Carmen,

Guzmán Ramírez Perla Marlene, Márquez Valdez Manuel Alejandro, Esponda Prado Juan Gerardo, Teniza Frías Eduardo

Hospital Ángeles Pedregal.

Introducción: El absceso cerebral bacteriano es una infección intracraneal relativamente rara. Entre los trastornos predisponentes están la otitis media, la mastoiditis, la sinusitis paranasal, el trauma craneoencefálico o los procedimientos neuroquirúrgicos. Objetivo: Ejemplificar el reto diagnóstico del absceso cerebral, ya que puede confundirse, logrando así retraso en el diagnóstico y tratamiento. Caso clínico: Masculino de 76 años, inicia con astenia, adinamia, bradilalia, bradipsiquia y disminución en la interacción al habla con familiares. A las 16:00 horas la familia lo nota con debilidad en las cuatro extremidades, afasia y agnosia de objetos. Se realiza resonancia magnética (RM) en la cual se observa lesión en región temporal izquierda sugestiva de absceso cerebral y datos de sinusitis. Se aísla Moraxella morgagni del líquido cefalorraquídeo, se mantiene con antibioticoterapia y debido a buena evolución se decide su egreso de la $\mathrm{UCl}$, ya que neurocirugía y otorrinolaringología no ofrecieron cirugía durante su estancia en la unidad. Discusión: El absceso cerebral se formó por propagación directa desde un foco de infección continuo (sinusitis paranasal).

\section{Metahemoglobinemia por azul de patente}

Teniza Frías Eduardo, Enríquez Barajas Adriana,

Carrillo Ramírez Silvia, Esponda Prado Juan Gerardo,

Hernández Cortés Christopher

Hospital Ángeles Pedregal. 
Introducción: Se ha descrito que diversos colorantes médicos son capaces de alterar las asociaciones de hemoglobina al oxígeno. Objetivo: Presentar un caso de metahemoglobinemia por azul de patente. Caso clínico: Femenino de 69 años con diagnóstico de colangiocarcinoma intrahepático, sometida a protocolo de estudio en el que se opta por colocación de bomba para quimioterapia intraarterial. Para la prueba de fuga del dispositivo se utilizó azul de patente, posterior a la administración de éste la paciente comenzó con desaturación de hasta $80 \%$ pese a fracción de oxígeno mayor a $60 \%$, se encontraron concentraciones de metahemoglobina de $3 \%$, hemodinámicamente estuvo estable durante el transoperatorio. A la emersión, pese a la reversión de agentes farmacológicos anestésicos, no recobró el estado de conciencia, además tuvo alteración en la mecánica ventilatoria, por lo que se reintubó y pasó a terapia intensiva donde fue vigilada durante 24 horas y remitió espontáneamente. Discusión: Ya se sabe que la administración de colorantes puede generar alteraciones a la concentración de metahemoglobina, pero es destacable que, pese a niveles de amonio normales, el colorante redujo significativamente el estado mental de la paciente.

\section{Confección entre COVID-19 y dengue: serie de casos}

Santos Macías Juan Enrique, Cavazos Obregón Oscar Humberto, Luviano García José Antonio, Sosa Medellín Miguel Ángel,

Almanza Valdez Héctor Edmundo

Hospital Clínica Nova.

En la actualidad, se ha visto un incremento en la incidencia de casos de dengue en el mundo, siendo éste un problema de salud pública de gran relevancia. Al día de hoy se han reportado 12,278 nuevos casos confirmados de dengue en México. El dengue se manifiesta con un espectro clínico muy amplio, resaltan síntomas como fiebre y cefalea. Desafortunadamente el mundo atraviesa una emergencia sanitaria: una pandemia causada por el virus SARS-CoV-2 que cursa con los mismos síntomas; por lo que éste es un reto diagnóstico y terapéutico. Cabe señalar que el presentar una coinfección traza un problema mayor ante el reto terapéutico que esto representa. A continuación, se presenta una serie de casos de coinfección entre SARS-CoV-2 y dengue, presentando una incidencia de $2.9 \%$ con un total de cinco casos, sin encontrar literatura mundial que hable al respecto. En nuestra serie de casos los pacientes fueron del sexo masculino, con una media de edad de 36 años, ingresaron al hospital a los 4.3 días en promedio de iniciado los síntomas, con cuadro inicial de fiebre, mioartralgias y cefalea, linfopenia y niveles de ferritina mayores a $10,000 \mathrm{ng} / \mathrm{mL}$ y PCR menores a $1 \mathrm{mg} / \mathrm{dL}$.

\section{Miedo a la intubación y reanimación} cardiopulmonar en población mexicana

Miranda-Ackerman Roberto Carlos, Ibarrola-Peña Juan Carlos, Almanza-Mena Yolanda Lorelei, Barbosa-Camacho Francisco José, González Ojeda Alejandro

Hospital San Javier.

Introducción: Un reto médico es lograr que los pacientes acepten planes terapéuticos que no logran comprender y que existen grandes barreras sociales y culturales hacia ellos. Objetivo: Identificar a la población mexicana que no acepta que se le practique reanimación cardiopulmonar (RCP) o un proceso de intubación y conocer los motivos que impulsan su decisión. Material y métodos: Estudio observacional. Encuestamos a 4,004 personas de entre 15 y 87 años. Investigamos su postura para recibir RCP o ser intubados y sus motivos para decidir no aceptar los procedimientos. Resultados: La edad promedio fue de $30.01 \pm 13.72$ años; $69.1 \%$ permitiría ser intubado y $87.5 \%$ permitiría RCP. Las mujeres y los adultos mayores de 50 años, así como los estados centro-sur del país, son los que mostraron mayor renuencia a estos procedimientos. Las razones más prevalentes para decidir no ser intubado ni recibir RCP son "considero es muy invasivo» y «no quiero alargar mi vida», respectivamente. Discusión: Los motivos más prevalentes denotan una falta de información sobre los procedimientos. Es responsabilidad de los médicos dar información oportuna y precisa para que el paciente pueda tomar una decisión fundamentada, así como respetar la decisión final del paciente.

qSOFA, SOFA y SAPS 3 como pronóstico de mortalidad en pacientes críticos atendidos en situación de escasos recursos: análisis retrospectivo de tres años en Piedras Negras, Coahuila, México

Rodríguez de Molina Serrano José Iván, * Hernández Pliego Rogelio Ramsés, ${ }^{\ddagger}$ Díaz Conde Marcelo§ ${ }^{*}$ Clínica México, HGZ 11, IMSS. Piedras Negras, Coahuila. ¥Instituto Politécnico Nacional, México. §Hospital General «Dr. Manuel Gea González».

Introducción: El acceso a la medicina crítica y la disponibilidad de recursos presenta retos en los sistemas de salud en el mundo. A pesar de lo anterior, la medicina crítica es requerida en sitios donde la infraestructura o el personal es escaso. El presente estudio se realizó en dos UTI de escasos recursos en Piedras Negras, Coahuila, México. Objetivo: Analizar la medición de qSOFA, SOFA y SAPS 3 como marcador predictor de mortalidad en los pacientes críticos atendidos en dos unidades de terapia intensiva con escasos recursos, en Piedras Negras, Coahuila, México. Material y métodos: Estudio retrospectivo. Dos unidades de terapia intensiva de escasos recursos en Piedras Negras, Coahuila, México, en pacientes críticos con edad > 18 años, atendidos de noviembre de 2016 a julio de 2019. Se recabaron las características demográficas, diagnósticos principales, días de estancia y mortalidad en la UTI y hospital, SOFA, qSOFA y SAPS 3. Resultados: En el periodo de estudio se atendieron 211 pacientes y se trasladaron 43 pacientes, predominó el sexo femenino con $52.4 \%$, la edad promedio fue de 46.4 años. El principal diagnóstico de ingreso fue sepsis en $34.9 \%$, la mortalidad en la UTI y hospitalaria fue de $16.7 \%$ y $27.4 \%$, respectivamente. Se realizaron curvas ROC con los tres indicadores para mortalidad hospitalaria y en UTI: SAPS 3 AUC $=0.85$ IC 95\% 0.79-0.90 y AUC $=0.80 \mathrm{IC} 95 \%$ 0.74-0.88. SOFA AUC $=0.84 \mathrm{IC} 95 \% 0.78-0.91 \mathrm{y}$ $A U C=0.79$ IC 95\% 0.79-087. qSOFA AUC = 0.77 IC 95\% 0.69-0.85 y $A \cup C=0.79$ IC 95\% 0.70-0.89. Conclusión: Las escalas qSOFA, SOFA y SAPS 3 demostraron tener una buena capacidad para predecir mortalidad hospitalaria y en la UTI, en pacientes críticos atendidos en situación de escasos recursos.

\section{Impacto en mortalidad a 28 días con} metilprednisolona en paciente COVID-19 crítico

Rivera-Chávez Manuel José, ${ }^{*}$ Londoño-Rodríguez Mauricio, ${ }^{\ddagger}$ Valero-Vidal Fernanda,§ Valero-Vidal Maximiliano, $§$

García-Pons José Fernando"

${ }^{*}$ Hospital Regional de Alta Especialidad del Bají (HRAEB).

¥Departamento de Anestesiología. SUniversidad del Valle de

México Campus Querétaro. "Universidad de Guanajuato.

La pandemia por SARS-CoV-2 (COVID-19) ha puesto en jaque a los sistemas de salud a nivel mundial, al momento del desarrollo del presente no existe ningún fármaco o vacuna disponible para mitigar los daños causados por esta novel enfermedad. Objetivo: Valorar la utilidad de la metilprednisolona para reducir la mortalidad a 28 días en pacientes COVID-19 graves, ingresados en la UCI de un hospital de tercer nivel de atención. Material y métodos: Población de pacientes mayores de 18 años, con diagnóstico de COVID-19 críticos, se administró dosis de metilprednisolona $80 \mathrm{mg}$ IV cada 12 horas por 10 días con posterior cambio a prednisona por tres días. Resultados: Trece pacientes (ocho hombres, cinco mujeres), edad promedio de 56 años. Diabetes mellitus (DM) (9), hipertensión arterial sistémica (HTAS) (6), mortalidad asociada a 28 días $7 \%$ (1), resto egresado a domicilio con supervivencia a 28 días de 100\% (12). Proporción mejoría de 92\%, IC 95\% (0.66-0.98). Discusión: El empleo de metilprednisolona sistémica probablemente reduce la mortalidad a 28 días en pacientes con COVID-19 críticos. 
Incidencia de elevación de enzimas hepáticas (EEH) en pacientes con COVID-19, relación con morbilidad y asociación con mortalidad en el Departamento de Medicina Crítica

Ángeles Márquez Mónica, Franco Granillo Juvenal, Aguirre Sánchez Janet

Centro Médico ABC. Ciudad de México, México.

Introducción: El Colegio Americano de Gastroenterología reportó enzimas hepáticas anormales como principal manifestación hepática en pacientes con COVID-19. Éstas son más prevalentes en enfermedades graves, por lo que se supone puede ser predictor de gravedad. Objetivo: Conocer la incidencia de anormalidades de EEH, comprobar relación con morbilidad y mortalidad. Material y métodos: Se realizó un estudio observacional, descriptivo en una cohorte de pacientes hospitalizados de marzo a abril de 2020. Se tomaron datos demográficos, escalas de gravedad, mortalidad y pruebas de funcionamiento hepático. Se utilizó t de Student, Wilcoxon y regresión de Cox con SPSS22. Resultados: Fueron 50 pacientes, promedio de edad de 57.8 años, de los cuales $76 \%$ (38) presentaron anormalidad de AST/ALT, se observó mayor incidencia de comorbilidades como diabetes mellitus (DM) $(p=0.01)$, hipertensión arterial sistémica (HAS) $(p=0.01)$, sobrepeso $(p=0.01)$ y tabaquismo $(p$ $=0.02$ ). La mortalidad fue de $13 \%$ en los pacientes con EEH y $8 \%$ en los pacientes sin EEH; sin embargo, no se encontró asociación estadística entre EEH y supervivencia $(p=0.31)$. Discusión: La incidencia en nuestro estudio coincide con el de la literatura reportada en pacientes críticos. La EEH se asoció con comorbilidades como DM, HAS, sobrepeso, obesidad y tabaquismo. La EEH no influyó en la supervivencia.

Prevalencia de hemorragia de tubo digestivo en pacientes con COVID-19 grave

Ugarte Martínez Paulina, Franco Granillo Juvenal,

Aguirre Sánchez Janet

Centro Médico ABC. Ciudad de México, México.

Introducción: La hemorragia de tubo digestivo es una complicación reportada en pacientes críticos con tasa de 4.5-8\%. Los pacientes con COVID-19 grave cuentan con más de dos factores de riesgo establecidos para el desarrollo de esta complicación. Objetivo: Conocer la prevalencia y características de la población con COVID-19 grave con hemorragia de tubo digestivo en la Unidad de Terapia Intensiva. Material y métodos: Es un estudio retrospectivo, descriptivo, de los pacientes con COVID-19 grave en terapia intensiva del 13 de marzo al 30 de septiembre del 2020, en los que se realizó estudio endoscópico por hemorragia digestiva, se utilizaron medidas de tendencia central para su descripción. Resultados: Fueron 357 pacientes. Se encontró prevalencia de $3.9 \%(n=14) ; 57 \%(n=8)$ de las hemorragias fue secundario a úlceras gástricas, con edad media de 63 años (42-81 años); $50 \%(n=7)$ tuvo dos o más comorbilidades, de los cuales, $85 \%$ tuvo tratamiento con esteroide $(n=12)$ y $71 \%(10)$ anticoagulación, 100\% tuvo profilaxis para las úlceras por estrés. Conclusión: La prevalencia en nuestra población fue menor a la reportada a nivel internacional, aunque este estudio es retrospectivo y la prevalencia real puede estar infraestimada. El desarrollo de úlceras por estrés fue mayor.
Mortalidad hospitalaria en pacientes atendidos en la Unidad de Terapia Intensiva de un Hospital Privado de Tercer Nivel con diagnóstico de intoxicación aguda grave: experiencia de 10 años

Pérez Martínez Pablo, López Márquez Santa, Rentería Díaz Faustino Javier, Martínez Zubieta Ricardo, Cerón Díaz Ulises Wilfrido

Hospital Español de México.

Introducción: En nuestro país, las intoxicaciones representan un importante problema de salud pública, puesto que requieren atención de los servicios de urgencias, de las cuales $14 \%$ de ellas necesitan hospitalización, y hasta $6 \%$ manejo en una Unidad de Cuidados Intensivos. Objetivo general: Determinar la mortalidad hospitalaria de los pacientes con diagnóstico de intoxicación aguda grave, atendidos en la Unidad de Terapia Intensiva del Hospital Español del 01 de septiembre de 2009 al 31 de marzo de 2020. Material y métodos: Estudio exploratorio, descriptivo y retrolectivo de 10 años en el que se incluyeron todos los pacientes con diagnóstico de intoxicación aguda grave atendidos en la Unidad de Cuidados Intensivos de un Hospital Privado de Tercer Nivel. Resultados: Se encontró un total de 50 pacientes que cumplieron con los criterios de inclusión para el estudio, se observó afección principal de hombres, con antecedentes psiquiátricos previos, el agente más frecuentemente encontrado fueron los fármacos y la mortalidad fue de $6 \%$. Conclusión: La mortalidad encontrada fue similar a la publicada en la literatura, a pesar de no usar terapia antidotal específica. Lo que refleja la importancia del soporte vital básico y avanzado en el paciente intoxicado ingresado en $\mathrm{UCl}$.

Lesión renal aguda durante la hospitalización como factor pronóstico de mortalidad en infección por SARS-CoV-2 y COVID-19 en pacientes de Terapia Intensiva del Hospital Regional PEMEX Ciudad Madero

González García Carlos Jesús, ${ }^{*}$ Ramírez Gutiérrez Álvaro Eduardo, ${ }^{\ddagger}$ Turrubiates Hernández Theno Alexandro, ${ }^{*}$

Sánchez Medina Jorge Rosendo§

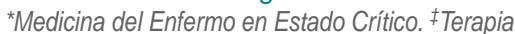

Intensiva. §Hospital Regional Ciudad Madero.

Introducción: Se ha descrito asociación de lesión renal aguda (LRA) y mortalidad en pacientes COVID-19. La incidencia varía entre series y países, la evidencia sugiere que afecta a $20 \%$ de los pacientes hospitalizados y más de $50 \%$ de los pacientes en terapia intensiva, con mayor mortalidad. Objetivo. Determinar la asociación de LRA y mortalidad durante la estancia en terapia intensiva. Material y métodos: Se estudiaron pacientes con diagnóstico confirmado de infección por SARS-CoV-2 mediante reacción en cadena de polimerasa (PCR) y que desarrollaron LRA durante cualquier momento de la hospitalización, ingresados a Unidad de Terapia Intensiva del Hospital Regional de Ciudad Madero del 01 de marzo al 01 de octubre de 2020. Resultados: La mortalidad fue de $56 \%$ para aquellos sin LRA, $50 \%$ para AKIN I, $66 \%$ para AKIN II y $83 \%$ para AKIN III. Hubo asociación de LRA y mortalidad con punto de corte creatinina $>1.2 \mathrm{mg} / \mathrm{dL}$. La mortalidad global para LRA fue de $60 \%$, sobre todo si aparece después de las 72 horas. Discusión: La presencia de LRA en pacientes con infección por SARS-CoV-2 es más común de lo reportado inicialmente y tiene asociación importante con mortalidad, con una tasa similar a la reportada en otras series. 